$$
\begin{aligned}
& \sum l^{2}=1, \sum l_{1}^{2}=1, \quad \frac{l_{1}}{a_{1}}=\frac{m_{1}}{b_{1}}=\frac{n_{1}}{c_{1}}=\frac{1}{\beta}, \\
&\left|\begin{array}{lll}
l & m & n \\
l_{1} & m_{1} & n_{1} \\
l_{11} & m_{11} & n_{11}
\end{array}\right|=U
\end{aligned}
$$

and the surface is defined by the equations

$$
\begin{aligned}
& x=a+k u v, \\
& y=b+\frac{k u v}{U \sqrt{1-k^{2}}}(\cos \psi+k \sin \psi U), \\
& z=c+\frac{k u v}{U \sqrt{1-k^{2}}}(\sin \psi-k U \cos \psi),
\end{aligned}
$$

where $a, b, c$ are perfectly determinate functions of $u$, and

$$
k=\frac{\alpha}{\sqrt{1+\alpha^{2}}}, \quad \sin k \psi=\frac{k u}{\sqrt{1-k^{2}}} .
$$

Bryn Mawr College, Penna.,

December, 1908.

\title{
NOTE ON ENRIQUES'S REVIEW OF THE FOUNDATIONS OF GEOMETRY.
}

\author{
BY MR. A. R. SCHWEITZER.
}

(Read before the Chicago Section of the American Mathematical Society, A pril 18, 1908.)

1. As is well known, a continuous descriptive space may be extended to a projective one by the suitable definition of the projective points. For the definition of euclidean geometry on the basis of descriptive-projective geometry, we distinguish between the proper and improper points of the space and the assumption is made that all the improper points lie on the unique improper plane. This assumption may be provided for by means of a 
suitable descriptive axiom. The following question then arises :* Is it possible to define a unique euclidean geometry on the basis of the preceding descriptive-projective space without introducing further indefinables or axioms? In the present note I show that such a definition is possible if we employ the notion of a propositional function. $\nmid$

2 . The conventional manner of defining euclidean geometry on the basis of descriptive-projective geometry is to select an elliptic polar system, say $\Sigma_{0}$, in the unique improper plane and to define perpendicularity, congruence, etc., with reference to it. Every such elliptic polar system $\Sigma_{0}$, however, gives rise to a euclidean geometry $G\left(\Sigma_{0}\right)$. A typical congruence proposition in this geometry will have the form : + "If . . . with reference to $\Sigma_{0}$, then . . . with reference to $\Sigma_{0}$." Thus the class of elliptic polar systems $\left\{\Sigma_{0}\right\}$ in the improper plane gives rise to a unique class of euclidean geometries $\left\{G\left(\Sigma_{0}\right)\right\}$. The members of this latter class are, however, given by means of the geometry function $G(\Sigma)$ of a variable elliptic polar system $\Sigma$ in the improper plane. This geometry function is then uniquely defined. A typical congruence proposition of $G(\Sigma)$ has the form : "If . . . with reference to $\Sigma$ then ... with reference to $\Sigma$." The terms of the latter implication are propositional functions of the variable $\Sigma$. As Russell maintains, $\S$ such terms are not propositions but yield propositions for every constant value of the variable; the implication which involves the propositional functions as terms is, however, a proposition and with reference to the implication, in the terminology of Peano, $\Sigma$ is an apparent variable.

\footnotetext{
* Compare Enriques, Encyklopädie der mathematischen Wissenschaften, vol. III, 1, p. 34 .

† See B. Russell, The Principles of Mathematics, pp. 13, 82.

The spaces indicated are to be filled out suitably by congruence properties.

\& l. c., p. 13 . In this connection, see some interesting points brought out by a controversy between McColl and Russell in the journal Mind, n. s. vol. 17 (1908), pp. 151-152; 300-302.
} 